\title{
Single Men Have a Higher Morbidity Risk of Acute Coronary Syndrome at a Younger Age than Married Men
}

\author{
Yasushi Watanabe $^{1)}$, Go Kimura ${ }^{1)}$, Shinsuke Taguchi ${ }^{1)}$, Yoshiki Uehara ${ }^{1)}$ and Yoshihiro Suematsu ${ }^{2)}$
}

\begin{abstract}
Introduction: In Japan, the prevalence of unmarried people is increasing. Sometimes, young men who suffer from acute coronary syndrome (ACS) are unmarried. Few reports discussing age at ACS onset and marital status have been reported. Methods and Results: We analyzed 160 patients who came to our hospital with a diagnosis of ACS between January 2017 and September 2018. We excluded 33 men who were over 75 years old. Among the remaining 99 men who were under the age of 75 years, we compared a married group (MG: $n=$ 66) and a single group (SG: $n=33$ ). The clinical characteristics were examined using $t$-tests. The age at the onset of ACS was significantly younger in the SG than in the MG $(62.6 \pm 8.6$ vs. $57.3 \pm 10.0$ years; $P=0.007)$. The low-density to high-density lipoprotein ratio $(\mathrm{L} / \mathrm{H})$ was significantly higher in the SG than in the MG (MG: $2.7 \pm 0.9$ vs. SG: $3.2 \pm 1.3 ; P=0.027$ ). Using statin was significantly higher in the MG than in the SG (MG: $18.3 \% \pm 34.2$ vs. SG: $6.0 \% \pm 40.1 ; P=0.038)$. There were no differences in hypertension, low density lipoprotein (LDL), high density lipoprotein (HDL), triglyceride, diabetes mellitus, $\mathrm{HbA}_{1 \mathrm{c}}$, body mass index (BMI), serum creatinine, uric acid and smoking habit between the two groups. There was no difference in the previous percutaneous coronary intervention (PCI), the percentage of ST elevation myocardial infarction (STEMI), peak creatinine kinase-MB (CK-MB), the number of significant fixed stenosis, the percentage of the culprit lesion was LAD (left anterior descending) artery. Multi-variable analysis revealed that marital status was the independent risk factor for young onset of ACS. Conclusion: Single men have a greater risk of early-onset ACS than married men. However, the mechanism for this difference remains unknown, and further studies are required.
\end{abstract}

KEY WORDS: ACS (acute coronary syndrome), early-onset, marital status, single men

\section{Introduction}

The average age at the time of marriage is increasing in Japan. In addition, the percentages of people who have remained single or who are divorced are increasing ${ }^{1}$. Consequently, the prevalence of single persons is expected to increase. Some studies have indicated that marital status is an important factor associated with mortality and cardiovascular diseases ${ }^{2-5}$. Thus, the status of Japan as a country with one of the greatest longevities in the world is at risk. However, few studies have investigated the relationship between the age of acute coronary syndrome (ACS) onset and marital status. We hypothesized that single men might have a greater risk of early-onset ACS and might have more coronary risk factors than married men. This study's purpose is to reveal that marital status could be one of the coronary risk factors

\footnotetext{
${ }^{1)}$ Department of Cardiology, Mito Brain Heart Center, Mito, Ibaraki Japan,

${ }^{2)}$ Department of Cardiovascular Surgery, Tsukuba Memorial Hospital, 1187-299 Kaname, Tsukuba, Ibaraki 310-0004, Japan

This paper was presented at $32^{\text {nd }}$ Annual meeting of the Japanese coronary association in Kumamoto (November 16 $6^{\text {th }}, 2018$ )

E-mail: suematsu@tf7.so-net.ne.jp

Received: July 2, 2019, Accepted: November 15, 2019

Doi: 10.7793/jcad.25.19-00010
}

or not.

\section{Methods}

This study is a retrospective observational study which search for the relationship between age of onset for ACS and marital status. This study examined a total of 160 consecutive patients (132 men and 28 women, aged 34 to 91 years) who visited our hospital (Mito Brain Heart Center) with a diagnosis of ACS between January 2017 and September 2018. ACS was diagnosed according to the appearance of the electrocardiogram (ECG) ST elevation myocardial infarction (STEMI), non-ST elevation myocardial infarction (NSTEMI), and unstable angina. Detailed diagnosis of ACS was based on ACS guideline of the Japanese Circulation Society ${ }^{6}$. All the patients were Japanese (Mongolian ancestry). We excluded 33 men who were over the age of 75 years, since many of these elderly patients had been recently widowed according to their partner death. The remaining 99 men who were under the age of 75 years men were then separated into a married group (MG: $n=66$ ) and a single group (SG: $n=$ 33) (Fig. 1). Only a legal connection was considered as a marital relation at the onset of ACS. Separated, divorced, widowed, never married, or common-law marriages were not taken into con- 


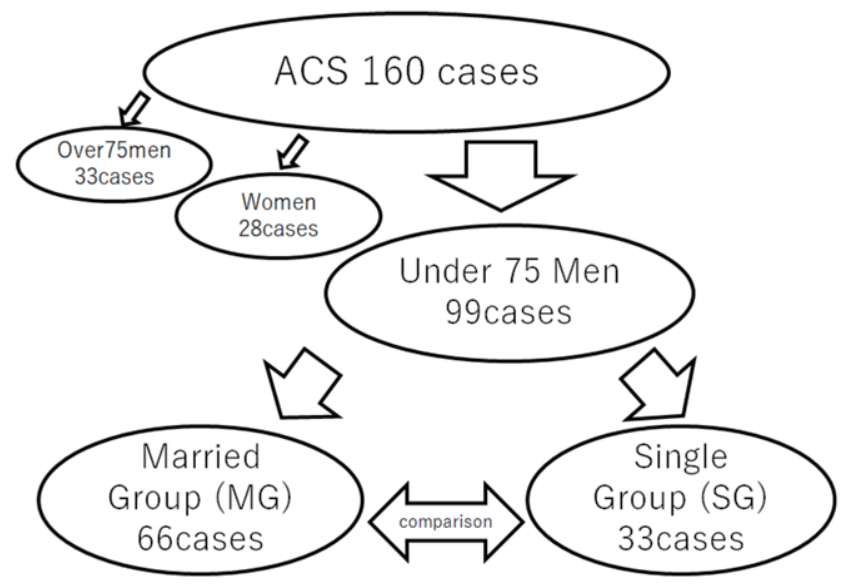

Fig. 1 Patients selection.

sideration. The subjects' perceptions of their marriage (good or bad) were not taken into consideration, either. We examined the clinical characteristics of the two groups. Clinical data including age of onset, hypertension, low density lipoprotein (LDL), high density lipoprotein (HDL), low-density to high-density lipoprotein ratio $(\mathrm{L} / \mathrm{H})$, triglyceride, using statin, diabetes mellitus, HbA1c, body mass index (BMI), serum creatinine, uric acid and smoking habit within 10 years. The history of administration for anti-hypertension medicine, the average systolic blood pressure (BP) over $140 \mathrm{mmHg}$ or diastolic BP over $90 \mathrm{mmHg}$ were diagnosed as hypertension. The $\mathrm{HbA}_{1 \mathrm{c}}$ over $6.5 \%$, random blood sugar lever over $200 \mathrm{mg} / \mathrm{dl}$ or fasting blood sugar level over $126 \mathrm{mg} /$ $\mathrm{dl}$ were diagnosed as diabetes mellitus. We also examined history of percutaneous coronary intervention (PCI), the percentage of STEMI in ACS, peak CK-MB (creatinine kinase-MB), the number of significant fixed stenosis, the percentage of the culprit lesion was LAD (left anterior descending) artery. The peak CKMB was measured every three hours after PCI. The maximum data was recorded as the peak CK-MB. Institutional review board approval was provided before publication of this article and reporting of the information. Informed consent was obtained by all patients.

\section{Statistical analysis}

These parameters were compared using $t$-tests. Statistical analysis continuous data were expressed as mean \pm standard deviation (range) and categoric variables were expressed by percentage. Statistical calculations were conducted with SPSS11.5 (SPSS Inc. Chicago, IL) software. Difference was considered statistically significant with a p value lower than 0.05 . Multivariate relative risk with $95 \%$ confidence intervals and odds ratio were also calculated.

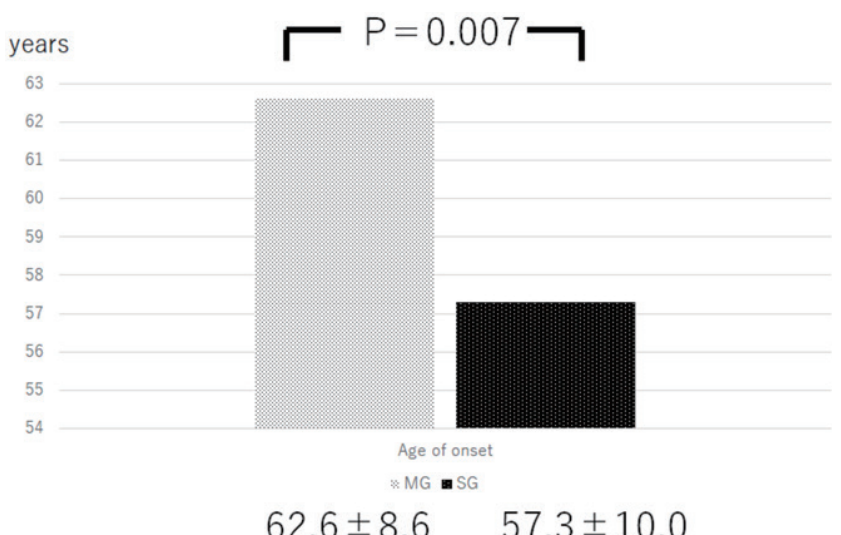

Fig. 2 Age at ACS onset.

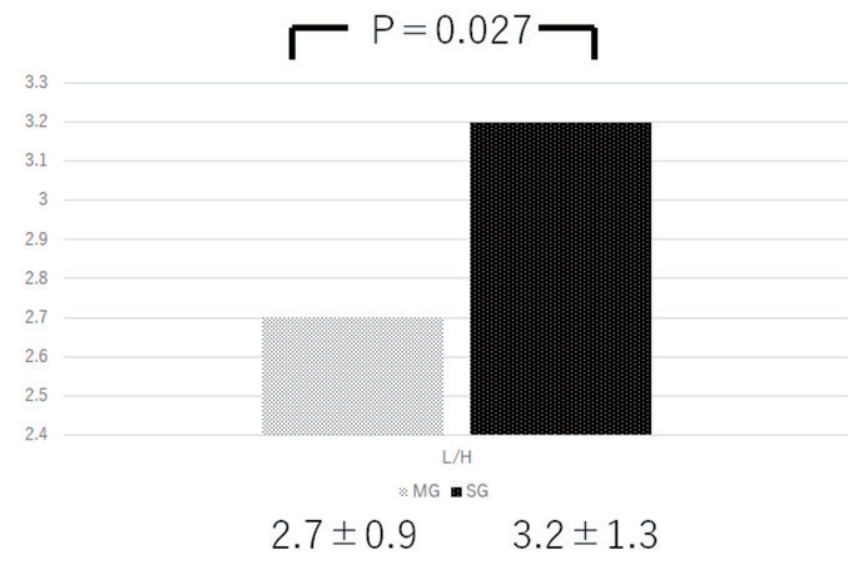

Fig. 3 The low-density to high-density lipoprotein ratio (L/H).

\section{Results}

The age at ACS onset was significantly younger in the SG than in the MG (MG: $62.6 \pm 8.6$ years vs. SG: $57.3 \pm 10.0$ years ; $P=0.007)$. This difference in age of onset was greater than 5 years (Fig. 2). L/H was significantly higher in the SG than in the MG (MG: $2.7 \pm 0.9$ vs. SG: $3.2 \pm 1.3 ; P=0.027$ ) (Fig. $3)$. Using statin was significantly higher in the $\mathrm{MG}$ than in the SG (MG: $18.3 \% \pm 34.2$ vs. SG: $6.0 \% \pm 40.1 ; P=0.038$ ). No significant differences in hypertension, LDL, HDL, triglyceride, diabetes mellitus, HbA1c, BMI, serum creatinine, uric acid and smoking habit were seen between the two groups. There was no difference in previous PCI, the percentage of STEMI, the level of peak CK-MB, the number of significant fixed stenosis, the percentage of the culprit lesion was LAD (Table 1). Multi-variable analysis revealed that marital status was the independent factor for younger onset of ACS ( $\mathrm{p}=0.047$, Odds ratio=0.371, confidence interval 0.139 to 0.986 ).

\section{Discussion}

In this analysis, our clinical impression that single males have 
an earlier onset of ACS was statistically demonstrated to be true However, very few differences in coronary risk factors were seen between married and single men. In our study, the $\mathrm{L} / \mathrm{H}$ ratio was higher in the SG, compared with the MG. Using statin was higher in the MG, compared with SG. We assumed that single men tended to eat unhealthy food more frequently than married men. If this were to be true, a larger number of differences in coronary risk parameters would have been expected. However, no significant differences in major risk factors for example hypertension, diabetes mellitus and smoking habit, were seen between the two groups. We did not take the quality of lipid intake, for example EPA/AA or apolipoprotein, into consideration in the present study, which might have affected the results. Married men may tend to be payed attention by their partner to take medicine, like statin.

From ancient times, it is generally said that loneliness feeling is not good for mental health. Many studies have suggested that social networks promote a longer life ${ }^{2,3)}$. Kaplan et al. reported that current marriage was associated with longer survival, and among the not-married categories, having never been married was the strongest predictor of premature mortality ${ }^{4}$. Marital status has been reported to be independently associated with cardiovascular outcome ${ }^{7)}$. A group from Greece reported that never-married ACS patients had a 2.8-times higher risk of dying during hospitalization than married patients, after adjusting for various confounders ${ }^{8}$. Chun et al. analyzed 34 studies containing more than two million participants. Compared with married participants, being unmarried (never married, divorced or widowed) was associated with an increased odds ratio (OR) of cardiovascular disease $(\mathrm{OR}=1.42)$. Single men and women with myocardial infarction reportedly had an increased mortality $(\mathrm{OR}=1.42)$ compared with married participants ${ }^{9)}$. A study performed in patients undergoing bypass surgery reported that married patients were 2.5 times more likely to be alive after 15 years compared with unmarried patients ${ }^{10)}$. Thus, there are some studies that marital status is good for health, especially to cardiovascular disease. Marita status will influence not only for onset of cardiovascular disease (CVD) but also prognosis after $\mathrm{CVD}^{10)}$.

Divorced and widowed men showed higher risks of mortality from cardiovascular disease compared with married men. Widowhood has been previously associated with increased risk of adverse cardiovascular events and mortality ${ }^{11-14)}$.

Although, there are a few differences between men and women, or among the countries. Women might not be affected by marital status, compared with men. If anything, marriage might increase the mortality risk for women. A group from the Netherlands reported that nonmarried men had significantly higher relative risks of 1.7 and 2.2 for all-cause mortality and coronary mortality, compared with married men ${ }^{15}$. A group of Sweden re- ported that marital stress worsened the prognosis of women with coronary heart disease ${ }^{16)}$. A British group reported that being single was associated with a higher mortality (hazard ratio $=1.45$ ), whereas being divorced and being widowed was not associated with an excess mortality risk (each hazard ratio $=1.09)^{17}$. A Japanese group reported that never-married men have higher mortality risks from cardiovascular disease (relative risk $[R R]=$ $3.05)$ and respiratory disease $(R R=2.43)$ after adjustments for potentially confounding variables, compared with married men. For never-married women, there was a smaller but significantly higher risk of mortality from all causes $(\mathrm{RR}=1.46)$, , but such a trend was not observed for women ${ }^{18)}$. Prior studies evaluating the effect of sex on cardiovascular outcomes in divorced individuals are conflicting. A large meta-analysis of 29 studies found that, compared with the married group, all-cause mortality was higher for both divorced men and women, with minimal difference between groups ${ }^{19)}$. Other studies have reported that men are at higher risk after divorce than their female counterparts ${ }^{2,5)}$ or that divorced women have higher risk compared with men ${ }^{20,21)}$.

In Japan, the percentage of people who are remaining single is increasing, and counterplans to promote marriage might become an important health policy. Marriage may limit the risk of cardiovascular disease, although the reason remains unknown. The cause might be psychological, but scientific investigations of this matter are difficult. We need to reconsider about the detail of life habit difference between married and single men. Further investigation is needed.

\section{Limitations}

Our study was performed retrospectively at a single center, and retrospective analysis. The number of cases were small. A larger number of cases and prospective analysis might have revealed further differences in risk factors between the two groups.

\section{Conclusion}

Single men have a greater risk of early-onset ACS than married men. The mechanism remains unknown, and further studies are needed.

\section{Conflicts of interest}

None.

\section{References}

1) Ministry of Health, laborer and Welfare of Japan. https: //www.mhlw. go.jp/english/database/db-hw/dl/vs06_1_Annual_Trends_in_Marriages.pdf (accessed date 2019-10-25)

2) Molloy GJ, Stamatakis E, Randall G, et al: Marital status, gender and cardiovascular mortality: behavioral, psychological distress and metabolic explanations. Soc Sci Med 2009; 69: 223-228

3) Berkman LF, Syme SL: Social networks, host resistance, and mor- 
tality: a nine-year follow-up study of Alameda County residents. Am J Epidemiol 1979; 109: 186-204

4) Kaplan RM, Kronick RG: Marital status and longevity in the United States population. J Epidemiol Community Health 2006; 60: 760765

5) Rogers RG: Marriage, sex, and mortality. J Marriage Fam 1995; 57: $515-526$

6) Kimura K, Ako J, Arai H, et al: JCS 2018 guideline on diagnosis and treatment of acute coronary syndrome. http: //www.j-circ.or.jp/guideline/pdf/JCS2018_kimura.pdf (accessed date 2019-11-13)

7) Schultz WM, Hayek SS, Tahhan AS, et al: Marital status and outcome in patients with cardiovascular disease. J Am Heart Assoc 2017; 6. doi: 10.1161/JAHA.117.005890

8) Panagiotakos DB, Pitsavos C, Kogias Y, et al: Marital status, depressive episodes, and short-term prognosis of patients with acute coronary syndrome: Greek study of acute coronary syndrome (GREECS). Neuropsychiatr Dis Treat 2008; 4: 425-432

9) Wong CW, Kwok CS, Narain A, et al: Marital status and risk of cardiovascular diseases: a systemic review and meta-analysis. Heart 2018; 104: 1937-1948

10) King KB, Reis HT: Marriage and long-term survival after coronary artery bypass grafting. Hearth Psychol 2012; 31: 55-62

11) Ben-Shlomo Y, Smith GD, Shipley M, et al: Magnitude and causes of mortality differences between married and unmarried men. J Epidemiol Community Health 1993; 47: 200-205

12) Va P, Yang WS, Nechuta $S$, et al: Marital status and mortality among middle age and elderly men and women in urban Shanghai. PLoS One 2011; 6: e26600
13) Williams BR, Zhang Y, Sawyer P, et al: Intrinsic association of widowhood with mortality in community-dwelling older women and men: findings from prospective propensity-matched population study. J Gerontol A Biol Sci Med Sci 2011; 66: 1360-1368

14) Mostofsky E, Maclure M, Sherwood JB, et al: Risk of acute myocardial infarction after the death of a significant person in one's life: the Determinants of Myocardial Infarction Onset Study. Circulation 2012; 125: 491-496

15) Mendes de Leon CF, Appels AW, Otten FW, et al: Risk of mortality and coronary heart disease by marital status in middle-aged men in The Netherlands. Int J Epidemiol 1992; 21: 460-466

16) Orth-Gomér K, Wamala SP, Horsten M, et al: Marital stress worsens prognosis in women with coronary heart disease: The Stockholm Female Coronary Risk Study. JAMA 2000; 284: 3008-3014

17) Cheung YB: Marital status and mortality in British women: a longitudinal study. Int J Epidemiol 2000; 29: 93-99

18) Ikeda A, Iso H, Toyoshima H, et al; JACC Study Group: Marital status and mortality among Japanese men and women: the Japan Collaborative Cohort Study. BMC Public Health 2007; 7: 73

19) Manzoli L, Villari P, M Pirone G, et al: Marital status and mortality in the elderly: a systematic review and meta-analysis. Soc Sci Med 2007; 64: 77-94

20) Johnson NJ, Backlund E, Sorlie PD, et al: Marita status and mortality: the national longitudinal mortality study. Ann Epidemiol 2000; 10: $224-238$

21) Kriegbaum M, Christensen U, Andersen PK, et al: Dose the association between broken partnership and first time myocardial infarction vary with time after break-up? Int J Epidemiol 2013; 42: 1811-1819 